\title{
Autonomic dystonia syndrome in conditions of failure of the Bauhinia valve and after its surgical correction as a possible link in the etiopathogenesis of peptic ulcer disease
}

Martynov Vladimir Leonidovich

Doctor of Medical Sciences, Associate Professor, National Research Nizhny Novgorod State University named after N.I. Lobachevsky (UNN), Russia. *Corresponding Author: Martynov Vladimir Leonidovich, Doctor of Medical Sciences, Associate Professor, National Research Nizhny Novgorod State University named after N.I. Lobachevsky (UNN), Russia.

Received Date: March 30, 2021; Accepted Date: April 07, 2021; Published Date; July 19,2021

Citation: Martynov V. Leonidovich. (2021) Autonomic dystonia syndrome in conditions of failure of the Bauhinia valve and after its surgical correction as a possible link in the etiopathogenesis of peptic ulcer disease. J. Surgi Case Repo and Imag. 4(5); DOI:10.31579/2690-1897/067

Copyright: ( 2021 Martynov Vladimir Leonidovich, This is an open-access article distributed under the terms of the Creative Commons Attribution License, which permits unrestricted use, distribution, and reproduction in any medium, provided the original author and source are credited.

\begin{abstract}
The classic works of I.P. Pavlov shows strong communication mechanisms between the two most important life-supporting systems of the body - the nervous and the digestive. The role of the autonomic nervous system (ANS) in the occurrence of acute gastroduodenal erosions and ulcers has been proven [1]. The flow of sympathetic impulses causes an excessive release of mediators (catecholamines), which leads to disruption of tissue trophism. An important role in ulceration belongs to the parasympathetic nervous system.

Keyword: autonomic nervous system; bauhinia valve; peptic ulcer disease
\end{abstract}

The classic works of I.P. Pavlov shows strong communication mechanisms between the two most important life-supporting systems of the body - the nervous and the digestive. The role of the autonomic nervous system (ANS) in the occurrence of acute gastroduodenal erosions and ulcers has been proven [1]. The flow of sympathetic impulses causes an excessive release of mediators (catecholamines), which leads to disruption of tissue trophism. An important role in ulceration belongs to the parasympathetic nervous system. Stress leads primarily to the stimulation of hypothalamic function. Stimulation of the anterior hypothalamus through parasympathetic pathways causes hypersecretion of hydrochloric acid and pepsin, hypermotility and hypertonicity of the stomach, and stimulation of the posterior hypothalamus, due to sympathetic impulses, leads to vasospasm and ischemia [2]. Dysfunction of the autonomic nervous system (NS) is considered as a pathogenetic factor in non-infectious gastrointestinal pathology $[3,4,5,6]$. The relationship between the VNS and gastroduodenal motility, which plays an important role in the development of gastroduodenal pathology (GDP), has been studied [7]. With hypermotility of the stomach and duodenum (Duodenum), the parasympathetic division of the ANS was noted, with hypomotorism - the sympathetic. The balance between the ANS sections is characteristic of the normomotor of the stomach and duodenum [8]. One of the reasons for the chronicity of the pathological process in the mucous membrane of the gastroduodenal zone (SD GDZ) are sphincter disorders, the manifestation of which is duodenogastric reflux (GDR), gastroesophageal reflux (GER). In the development of sphincter disorders, the priority role is given to the ANS dysfunction. A relationship was established between the state of the parasympathetic division of the
ANS and the presence of GHD in patients with chronic gastroduodenitis (CGD). Samarina O.V. [9] showed that in patients withsphincter disorders, hypersympathicotonic VR and insufficient autonomic support of activity (VOD) prevail.

In the pathogenesis of CGDP, an important role is assigned to the functioning of the ANS, because it has been proved that CGDP arises as a result of depletion of protective, adaptive mechanisms [7, 10], as a possible mechanism for the formation of GDP, the prevalence of parasympathetic regulation is considered, leading to a violation of the secretory-motor function of the stomach. Violation of the trophism of the coolant is explained by a decrease in the activity of the sympathetic link of the ANS. In the study of the state of the ANS in chronic gastroduodenal pathology (CGDP) (chronic gastroduodenitis, gastric ulcer, duodenal ulcer - CGD, UBZH, UB duodenal ulcer), contradictory results were obtained. When studying the state of the ANS in patients with ulcer, ambiguous results were also obtained. Some authors point to the predominance of the parasympathetic division of the ANS [11, 12], others - the sympathetic [13]. So, according to T.Yu. Kravtsova. et al. 1993 [13], in ulcer duodenal ulcer, the predominance of parasympathetic influences is noted, there is a reduced VR and insufficient VOD. Solovieva VG, [14] revealed a significant predominance of the sympathetic component of the ANS (there were the majority of such patients - 50-53\%), but some patients had a parasympathetic and normal ANS tone. According to V.L. Dmitriev. [15], among patients with duodenal ulcer, patients with eutonia predominate. There was no statistically significant difference between the number of patients with vagotonia and sympathotonia. According to O.V. 
Kokuev. [16], the duodenal ulcer is characterized by hypervagotonia, while the stomach tone, as a rule, is increased, the peristalsis is increased. Such movement disorders are usually combined with hyperacidity and increased enzymatic activity of gastric juice. With mediogastric ulcer, the vagus tone is reduced, the tone of the sympathetic part of the ANS predominates, as a result of which both tonic and peristaltic waves of the stomach decrease, the pyloric closure function is insufficient, which causes the development of GHD [15, 16]. Zimmerman Ya.S. et al. [17] found that in the period of exacerbation of ulcer, the reactivity of the sympathetic nervous system increases, but its tone remains within normal limits. Thus, the concentration of catecholamines in the blood was significantly higher than the norm, and their daily urinary excretion did not exceed the norm, the level in the gastric juice on an empty stomach was moderately increased. These shifts indicate a violation of the adaptive trophic function of the sympathetic nervous system in patients with peptic ulcer. Revealed the dependence of the vegetative tone on the timing of ulcer scarring and the course of the disease [18]. According to the results of the study, with a favorable course of the disease (without complications, with a scarring period of up to a month), the mobilization of defense mechanisms occurs, including an increase in the activity of the sympathetic link of the ANS and an increase in adrenergic influences on metabolic processes. In an unfavorable course (with complications, frequent relapses), but with a scarring period of up to 1 month, an increase in the ergotropic system was revealed, the use of still remaining reserves, and with long-term non-scarring ulcers, depletion of regulatory mechanisms is observed, an inability to provide an adequate response of the body, which, according to the author, is the reason for the enhancement of the function of the trophotropic system [18]. The dependence of VR and VOD on the duration of the disease and the severity of clinical symptoms was revealed.

In the pathogenesis of ulcer, great importance is attached to hemodynamic disorders, especially microcirculatory disorders. Dmitriev V.L. and Dudnikova E.V. [19, 12] found that patients with ulcer have microcirculation disorders of varying severity, the depth of which depends on the state of the ANS. The most pronounced microcirculatory disorders were in patients with a predominance of the sympathetic nervous system, and the least pronounced in patients with eutonia. An increase in acid production and proteolytic activity of gastric juice, a decrease in glycoproteins of gastric mucus, which occurs during an exacerbation of CGDP [20], was found in all types of ANS with the greatest changes in vagotonics [12]. A change in the acid-producing function of the stomach and the proteolytic activity of gastric juice towards aggression is the result of a violation of the conduction of the sympathetic nerves due to compression or infringement of them in the intervertebral foramen of 7-9 thoracic vertebrae. At the same time, basal acid production and proteolytic activity of gastric juice increase due to increased tone of parasympathetic NS [21]. A chronic inflammatory process that develops in the system of a whole organism causes disturbances in the nervous system. This circumstance allows us to classify the pathology of the digestive system as psychosomatic [22, $23,24]$. The link connecting mental and visceral manifestations within the framework of psychosomatic pathology is the ANS [25, 26]. Thus, the presented literature data indicate the important role of VNS dysfunction in the formation of pathology of the digestive system. Gastroduodenal erosions constitute the largest, most diverse and widespread group of pathological processes occurring mainly in patients of the youngest and most working age $[27,28]$. Despite the antisecretory drugs and antiHelicobacter pylori that are widely used in the treatment of erosions, a clear dynamics of the growth of erosive lesions and their hemorrhagic complications can be traced in recent studies [29, 30].

The influence of the autonomic nervous system (ANS) on the mechanisms of the implementation of pathological changes in the stomach in patients with gastroduodenal erosions is of particular interest, since the disorganization of neurotrophic control in the body of patients can lead to the formation of disorders of secretion and motility [31]. Modern medicine makes it possible to use scientifically based tests in determining the functional state of the ANS, in particular, the method of cardiorhythmography, based on the analysis of the structure of sinus heart rhythm, which opens up an opportunity for further study of this problem. the effectiveness of their treatment [32]. In the study of the ANS in patients with acute erosions, a predominantly sympathetic orientation of suprasegmental autonomic disorders was noted in the form of a predominance of the sympathetic type of autonomic tone $(47 \%$ of patients, $\mathrm{p}<0.01$ ). The secretory function of the stomach was mainly reduced $(\mathrm{pH}$ in the antrum of the stomach $7.6 \pm 0.4)$ and was combined with a hypokinetic type of motility $(0.12 \pm 0.005 \mathrm{mV}, \mathrm{p}<0.05)$ in $88.2 \%$ of patients. The study of the suprasegmental autonomic apparatus in patients with exacerbation of chronic erosive gastroduodenitis revealed the dominance of parasympathicotonia $(60.8 \%$ of patients, $p<0.01)$, sympathicotonia was noted in $17.4 \%$ of cases, the prevalence of eutonia was in $21.7 \%$ of patients. Treatment of patients with gastroduodenal erosions and ulcer should be carried out differentially, depending on the prevalence of clinical symptoms and the state of the ANS. Thus, the inclusion in the complex treatment of gastroduodenal erosions of correction of vegetative dysfunctions, which affects the main links of the pathogenesis of the disease, led to a distinct clinical improvement in the condition of patients, complete elimination of pain syndrome, and a reduction in the period of epithelialization of erosions by 5-6 days in $72.5 \%$ of patients [32].

The prevalence of irritable bowel syndrome (IBS) in the population varies on average from 10 to $25 \%$ (in the world $-11.2 \%$ ), and $2 / 3$ of patients with IBS are women [33, 34]. Inflammation in the intestinal wall forms visceral hypersensitivity, which provides a local symptom complex [35, 36]. The revealed quantitative and qualitative changes are nothing more than signs of bacterial overgrowth syndrome (SIBO). The leading role of SIBO in the pathogenesis of IBS can be judged by analyzing the data on the effectiveness of antibacterial and probiotic therapy with SIBO. Looking at IBS as a biopsychosocial suffering, one can distinguish the interaction of psychological factors, autonomic dysfunctions and motor disorders of various parts of the gastrointestinal tract [37]. As noted, the concept of "microbiota - intestine - brain" is actively used to explain the pathogenesis of chronic intestinal lesions [38]. The hypothesis that arose in connection with this about the involvement of the enteric nervous system in the subclinical chronic inflammatory process was first confirmed in the experiment [39]. Thus, excessive infiltration of the nerve plexuses of the muscular membrane with lymphocytes was revealed in patients with IBS with a predominance of diarrhea and severe refractory symptoms [38]. The described processes affecting the visceral (VNS) and central nervous systems (CNS), and their interaction, lead to an imbalance in the autonomic status in patients with IBS. In connective tissue dysplasia (CTD), autonomic dysfunction syndrome (SVD) is observed according to some data in $45-89 \%$ of cases [40]. It is argued that sympathicotonia is an "obligate" sign of CTD [40]. Gastroenterological manifestations of autonomic dysfunction in CTD have been studied insufficiently [41]. It is important that the predominance of the tone of the sympathetic division of the ANS is obligatory accompanied by a failure of the function of the 
sphincter apparatus of the gastrointestinal tract, with the development of various refluxes [9]. Evaluating the autonomic status in individuals with CTD using cardiointervalography (CIG), the authors registered a violation of autonomic tone in $65 \%$ of patients. Against the background of DST, sympathicotonia was more often registered. A.A. Semenkin [41] comes to the conclusion that in patients with DST there is a tension of the functional reserve of the regulation system from the ANS, which can be considered as predictors of the tension of the adaptive capabilities of the organism. The predominance of sensory neurons in the innervation of the ileocecal region indicates that the ileocecal valve is a highly sensitive formation of the human intestine [42, 43].

I.B. Bondarev [44] found from two to six nerve trunks, which had a direction from the ileocecal nerves to the anterior wall of the pyloric section of the stomach, near its border with the duodenum. Here, on the anterior wall of the pyloric stomach, the ileocecal nerves form a plexus that continues into the nerve plexuses of the duodenum and pancreas. Similar neural connections between the ileocecal region of the intestine, stomach, duodenum, pancreas are described by N. A. Bakeeva. SS Yudin [45] also pointed out the close connection of the ileocecal region with the stomach and duodenum. K.M. Bykov [46] emphasizes the connection between the ileocecal angle and the secretory and motor activity of the stomach through the nervous system. I.I. Grekov [47] considered chronic appendicitis as a provocateur of duodenal and gastric ulcers. We carried out a morphological study of preparations of the mucous membrane of the colon and ileum to identify possible inflammation, which may be a zone of pathological impulses for the formation of SVD [48]. During endoscopic examination (sigmoidoscopy, fibrocolonoscopy), a biopsy specimen of the mucous membranes of the rectum and colon was taken. The studies were performed before the operation and one year after it during the control examination. The study of biopsies of the colon mucosa in NBZ made it possible to establish various degrees of inflammation with infiltration by lymphoid cells (96\%), histiocytes (46\%) and plasma cells (28\%). The phenomena of edema occurred in $92 \%$ of studies, fibrosis - in $65 \%$. In addition, hemorrhages $(20 \%)$ and proliferation of intestinal epithelium (27\%) were found. The normal structure of the colon mucosa was observed only in $12 \%$ of cases.

A histological examination of the colon mucosa in 24 patients before surgery, as well as a year or more after it, revealed a decrease in the inflammatory process (biopsies were taken during fibrocolonoscopy and sigmoidoscopy). Sclerosis and fibrosis of the submucosal layer was registered in 9 patients, before surgery it occurred in 16 patients $(\mathrm{p}=$ 0.041 ). Proliferation of the epithelium of the colon mucosa after the operation took place in 7 , while before the operation - in 6 examined ( $p=$ 0.5 ). Plasma cell infiltration was noted in 1 patient, while before surgery it was observed in $6(\mathrm{p}=0.049)$. Edema was found in 2 patients, and before surgery it was observed in 22 ( $p$ <0.001). Infiltration with lymphoid cells was detected in 8 patients, and before surgery it was observed in 23 patients $(\mathrm{p}<0.001)$. The normal structure of the mucous membrane was found in 13 patients, whereas before the operation only in 3 ( $p=0.025)$. The data obtained served as the basis for the study of the state of the ANS in patients with NBD. We formulated the goal of this study: to determine the IBS-like syndrome, autonomic dystonia syndrome and connective tissue dysplasia in patients with Bauhinia valve insufficiency (NBZ) and after its surgical correction. Material and methods. The study included 215 patients (76 men and 139 women, age $18-70$ years, $\mathrm{m}=41.25\} 1.8$ years) with Bauhinia valve insufficiency, which was identified based on the results of irrigoscopy. IBS-like syndrome was diagnosed based on the III Rome criteria (Drossman D.A.
2006). Insufficiency of the Bauhinia valve and ineffectiveness of longterm conservative therapy of enterocolitic syndrome were indications for surgery. Surgical intervention consisted in the creation of an autonomously working areflux system in the zone of the ileocecal transition (RF patent No. 2225173).

Autonomic dystonia syndrome was assessed on the basis of complaints and cardiointervalography (CIG) data with NBZ and after its surgical correction. We proceeded from the statement that the duration of each specific R-R interval is a universal resultant response of the body to many external and internal influences. This method was used to assess the initial background vegetative status. For a correct assessment of heart rate variability (HRV), the following conditions were met: the examination was carried out no earlier than 1.5-2 hours after a meal; the room was darkened and the temperature in it was between 18 and 24 degrees Celsius; the factors leading to emotional arousal were absent; on the eve of the study, physiotherapy and drug treatment were canceled; the study was carried out with calm breathing; an adaptation period was organized before the study for 10 - 15 minutes; examination of women was carried out in the intermenstrual period. Thus, conditions were created for conducting a background test. The ECG was recorded for 10-15 minutes. For HRV analysis, conventional computerized electrocardiographs recording and analyzing a conventional electrocardiographic curve in real time, equipped with a HRV analysis program, were used. A finger heart rate monitor was placed on the second finger of the subject's left hand. The initial vegetative status was assessed using a photoplethysmographic heart rate analyzer with a computer program by R.M. Baevsky on the "Optim-510" apparatus

\section{Results and its discussion}

In all 215 patients, according to the results of irrigoscopy, the insufficiency of the Bauhinia valve was confirmed. Autonomic dystonia syndrome was detected in $92 \%$ of patients with NBD. Clinically, SVD was manifested by increased fatigue (92\%), dizziness (72\%), palpitations $(65 \%)$, headaches $(74 \%)$, hyperhidrosis $(66 \%)$, vasomotor lability $(66 \%)$. The vegetative tone of most patients changed towards sympathicotonia in $98 \%$ of patients. At the same time, $57 \%$ showed a moderate increase in sympathetic tone, $20 \%$ - a pronounced increase. Only $2 \%$ had a predominance of the parasympathetic tone of the autonomic nervous system. The data obtained confirm the hypothesis formulated by O.V. Samarina. [9] that the ANS sympathicotonia accompanies the insufficiency of the sphincters of the gastrointestinal tract (GIT). In this regard, the question of the need to determine the vegetative status for the purpose of screening for reflux disease sounds quite relevant. In terms of up to 1 year after bauginoplasty (BP), the disappearance of SVD was determined in $66 \%$ of patients (Wilcoxon's area, $p=0.001$ ), in $17 \%$ of patients, a decrease in SVD was noted, and in 17\% SVD was not eliminated. The feeling of palpitations disappeared in $80 \%$ (Wilcoxon cr., $\mathrm{P}=0.03$ ), It was observed in $65 \%$, dizziness was not noted in $74 \%$ (Wilcoxon's area, $\mathrm{p}=0.05$ ), dizziness decreased in $16 \%$, darkening in the eyes was not noted by $76 \%$, this complaint became less pronounced in $16 \%$ of patients. Interesting results of the study of gastric juice before and after bauginoplasty in 43 patients. In our opinion, they depend on the state of the ANS. After the operation, the normal state was revealed in 15 patients, while before the operation it was in $6(\mathrm{p}=0.022)$. The hypoacid state after surgery was determined in 4 patients, while before surgery - in $9(\mathrm{p}=0.114)$. Anacid state after surgery was noted in 8 , while before surgery - in $14(\mathrm{p}=0.108)$. 
The number of patients with a hyperacid state of gastric juice after surgery slightly increased from 15 to $16(p=0.5)$. The presence of bile in the gastric contents was registered in the same patients in whom it had occurred before the operation - in $16(\mathrm{p}=0.588)$. When sowing the contents of the stomach taken on an empty stomach, in 18 after NBZ correction, the growth of intestinal microflora was obtained in 6 cases, while before the operation in the same patients microbes were sown in 12 cases $(p=0.047)$. Thus, it was revealed that after the correction of only one link of reflux disease, namely NBZ, the acidity of gastric juice normalizes in $22 \%$ of patients ( 9 out of 43 patients $-p=0.22$ ) and the persistence of intestinal microflora in the stomach decreases by 2 times. The latter also confirms the role of NBZ in the development of the ascending route of gastrointestinal tract infection, and NBZ correction as a way to eliminate it. Bauginoplasty alone, according to the analysis of gastric juice, does not affect the elimination of GDR, as an integral part of RB. Conclusion. Cecoileal reflux arising in NBZ creates conditions for disruption of the microbiota of the small (SIBO) and colon (dysbiosis) intestines, which leads to permanent sluggish inflammation in the intestinal mucosa, which is indirectly confirmed by the presence of IBSlike syndrome in such patients. Further development of the pathological process, expressed in the concept of "microbiota-intestine-brain", disrupts the regulation and interaction between the ANS and the central nervous system. There is dystonia of the ANS with a predominance of sympathicotonia, which can be explained by disturbances in the metabolism of serotonin, the obligate precursor of which is trypotophan, and the coenzyme of tryptophan-5-monooxygenase is vitamin C. increased consumption and destruction of essential amino acids and vitamins by xenoflora, elimination of vitamin $C$ producers - bifidobacteria in the small intestine in conditions of the emerging syndrome of bacterial overgrowth. Timely diagnosis of Bauhinia valve insufficiency and its etiopathogenetic treatment, which may be surgical correction, allow interrupting the pathological cascade in the body in patients with NBD, normalizing the ANS function, which will exclude some links in the etiopathogenesis of peptic ulcer disease.

\section{Reference}

1. Dudnikova, E.V. (2001). The role of the autonomic nervous system in the pathology of the gastrointestinal tract. SouthRussian medical journal; 5-6:29-33.

2. Khokhola, V.P. (1983). On the etiology and pathogenesis of acute gastroduodenal ulcers; 2:68-72.

3. Wayne, A.M. (1971).Vegetative-vascular paroxysms / A.M. Wayne. M: Medicine.

4. Dulkin, L.A. (1991). Differentiation of the appointment of sedatives and psychoanaleptics for gastroenterological pathology in children, depending on the type of autonomic dysfunctions / L.A. Dulkin et al. // Question protection of motherhood and childhood ; P. 72-73.

5. Kuberger, M.B. (1989). The state of the autonomic nervous system during psycho-emotional load in children with noninfectious gastrointestinal pathology; 34:8. 15-17.

6. Filippov, G.P. (1993). Features of lipid metabolism and autonomic reactivity in people with pathology of the digestive system. Topical issues of cardiology.

7. S. 120-122. 7. Maltsev S.V. (1996). Features of the psychovegetative state in chronic gastroduodenitis in children of older genital age. Pediatrics; 4,38-42.

8. Reshetilov, Yu.I. (1990). The state of the autonomic nervous system and gastroduodenal motility. 61-64.

9. Samarina, O. V. (1996). Clinical significance of neurovegetative and psychoemotional changes in children with gastroduodenitis and combined sphincter disorders to substantiate treatment tactics: author. dis ... candidate of medical sciences - Moscow, .-- 23 p.

10. Tyurina, N.S. (1991). The functional state of the autonomic nervous system in children with chronic gastroduodenal pathology. Pediatrics; 4.24-28.

11. Volkova, T.A. (1995). Influence of the type of vegetative response on the nature of gastroduodenal pathology . Questions of Pediatrics; 74.

12. Dudnikova E.V. (1991). The role of the autonomic nervous system and factors of aggression and protection in the pathogenesis of chronic gastroduodenal pathology in children at the beginning of puberty: dis .... doc. Med. Sciences; $258 \mathrm{p}$.

13. Kravtsova, T.Yu. (1993). Psychovegetative syndrome in patients with duodenal ulcer and its correction by magnetopuncture with an alternating magnetic field. $J$. of Neurology and Psychiatry;. 93: 6. 50-52.

14. Solovyova, V.G. (1996) Altramet as a modulator of autonomic function support in gastric ulcer and duodenal ulcer; Problems of Experimental and Clinical Medicine. - Tomsk. Issue. 1. S. 58-60.

15. Dmitriev, V.L. (1990) Mechanisms of damage to the mucous barrier in peptic ulcer disease and their drug correction.

16. Kokueva, O. V., Korochanskaya N. (1996). In. Comparative characteristics of the personal characteristics of patients with gastric ulcer and duodenal ulcer. pp. 69-71.

17. Zimmerman, Ya.S. (1996) Topical issues of the pathogenesis and treatment of gastric ulcer / Ya.S. Zimmeran et al. // Stomach ulcer. Krasnodar, 159-160.

18. Burma, O.B. (1996) Chronobiorhythmological assessment of the autonomic nervous system in patients with duodenal ulcer with unfavorable course of the disease depending on the terms of ulcer scarring / O.B. Burma // Stomach ulcer, Krasnodar, pp. 24-26.

19. Dmitriev, V.L. (1990) Mechanisms of damage to the mucous barrier in peptic ulcer disease and their drug correction: Author's abstract. dis. cand. honey. sciences, Kharkov, $20 \mathrm{p}$.

20. Zakomerny, A.G. (1992) Modern clinical and epidemiological features of peptic ulcer disease in childhood and approaches to staged treatment of patients: Author's abstract. dis cand. honey. sciences. Moscow, $63 \mathrm{p}$.

21. Voinitsky, A.N. (1997) The role of the sympathetic nervous system in the pathogenesis of peptic ulcers of the gastrointestinal fistula: author. dis . cand. honey. sciences. St. Petersburg, $19 \mathrm{p}$.

22. Volkov, B.C., (1996) The influence of mental disorders on the course of peptic ulcer / V.S. Volkov, L.E. Smirnova // Klin. honey. T. 74. - No. 6. - P. 81.

23. Zaprudnov, A.M. (1997) Clinical and pathogenetic aspects of pharmacotherapy in pediatric gastroenterology / A.M. Zaprudnov // Pediatrics. - No. 6. - S. 30-35.

24. Mironychev, G. (1997) Psychosomatic aspects of peptic ulcer/ G. Mironychev et al. // Doctor. No. 6. - S. 13-15.

25. Zhilyaev, AG, (1999) Peculiarities of neurotic disorders in patients with forms of somatic diseases resistant to therapy. Zhilyaev, I.E. Zamakova // Kazan honey. Journal. No. 5. - S. 369-370.

26. Shvarkov, S. B. (1991) The use of express methods for the diagnosis of autonomic dystonia for the child population of Azerbaijan / S.B. Shvarkov, T.R. Talyshinsky // Azerb. honey. magazine - No. 3. - S. 17-23.

27. Kuznetsov, E.P. (2007) "Complete" erosion and hyperplastic polyps of the stomach - two sides of the same coin / E.P. 
Kuznetsov // Russian journal of gastroenterology, hepatology, coloproctology. No. 5, Appendix No. 30. - P. 142.

28. Nikolsky, V.I. (2009) Etiology and pathogenesis of acute gastroduodenal ulceration complicated by bleeding (literature review) / V.I. Nikolsky, K.I. Sergatsky // Bulletin of surgical gastroenterology. No. 4. - S. 53-63.

29. Nikiforov, P.A. (2007) "Chronic" erosion of the antrum of the stomach and their place among some diseases of the digestive system. Nikiforov and Sovt. // Russian journal of gastroenterology, hepatology, coloproctology. No. 5, Appendix No. 30. - P. 144.

30. Svintsitsky, A. S. Stomach erosion: questions of pathogenesis, clinical picture, diagnosis and treatment / A.S. Svintsitsky, G.A. Solovyova // Clinical Medicine. No. 9. - S. 18-23.

31. Komarov, F.I. (2010) Guide to gastroenterology / F.I. Komarov. M .: LLC "Medical Information Agency", 864 p.

32. Antonyan, V.V. (2010) Features of the autonomic nervous system in patients with gastric ulcer and duodenal ulcer / V.V. Antonyan et al. // Astrakhan Medical Journal. S. 29-33.

33. Hungin, P. (2005) Irritable bowel syndrome in the United States: prevalence, symptom patterns and impact / P. Hungin et al. // Aliment. Pharmacol. Ther. Vol. 21 (11). - P. 1365-1375.

34. Lovell, M. (2012) Global prevalence of and risk factors for irritable bowel syndrome: a meta-analysis / M. Lovell, C. Ford // Clin. Gastroenterol. Hepatol. Vol. 10 (7). - P. 712-721.

35. Sitkin, S.I. (2014) Inflammation, microbiota, visceral hypersensitivity - new and 'old' therapeutic targets in irritable bowel syndrome / S.I. Sitkin // Clinical perspectives of gastroenterology, hepatology. No. 3. - S. 43-52.

36. Kanazawa, M. (2011) Visceral hypersensitivity in irritable bowel syndrome / M. Kanazawa et al. // J. Gastroenterol. Hepatol. Vol. 26 (3). - P. 119-121.

37. Drossman, D.A. (2006) The functional gastrointestinal disorders and the Rome III process / D.A. Drossman // Gastroenterology. Vol. 130. - P. 1377-1390.
38. Tornblom, H. (2002) Fullthickness biopsy of the jejunum reveals inflammation and enteric neuropathy in irritable bowel syndrome / H. Tornblom et al. // Gastroenterology. Vol. 123 (6). - P. 1972-1999.

39. Laird, J.M. (2001) Responses of rat spinal neurons to distension of inflamed colon: role of tachykinin NK2 receptors / J.M., Laird et al. // Neuropharmacology. Vol. 40 (5). - P. 696-701.

40. Druk, I.V. (2004) Bronchial asthma associated with connective tissue dysplasia: features of the course of the disease: author. diss. ... Cand. honey. sciences. Omsk, 204 p.

41. Semenkin, A.A. (2011) Gastrointestinal manifestations in persons with connective tissue dysplasia associated with autonomic dysfunction syndrome [electronic resource] / A.A. Semenkin et al. // Attending doctor. No. 6.

42. Goncharov, P.P. (1941) On visceral reflexes from the intestine: author. dis ... cand. honey. Sciences: 14.00.06 / P.P. Goncharov, Moscow, 30s.

43. Bargen, I.A. (1940) Studies on ileocecai function (ileocecus)/ I.A. Bargen, H.R. Wesson, R.I. Iackian // Jurg.Jyn. a. Obst. Vol.71. - P.33.

44. Maksimenkov, A.N. (1972) Surgical anatomy of the abdomen / A.N. Maksimenkov. -66. L .: Medicine, S. 530 - 576.

45. Yudin, S.S. (1965) Sketches of gastric surgery / S.S. Yudin. M .: Medicine, $268 \mathrm{p}$.

46. Bykov, K.M. (1954) The cerebral cortex and internal organs / K.M. Bykov. - M .: Medicine, S. 416.

47. Grekov, I.I. (1952) Selected Works / I.I. Grekov. - M .: Medicine. S. 28-51.

48. Martynov, V.L. (2006) Refluxes of the digestive tract and their surgical correction: author. diss. .doct. honey. Sciences: 14.00.27 / Martynov Vladimir Leonidovich. Saransk, 261 p. 